\title{
ON THE MODULUS OF CONTINUITY OF MAPPINGS BETWEEN EUCLIDEAN SPACES
}

\author{
DIEUDONNÉ AGBOR* and JAN BOMAN
}

\begin{abstract}
Let $f$ be a function from $\mathrm{R}^{p}$ to $\mathrm{R}^{q}$ and let $\Lambda$ be a finite set of pairs $(\theta, \eta) \in \mathrm{R}^{p} \times \mathrm{R}^{q}$. Assume that the real-valued function $\langle\eta, f(x)\rangle$ is Lipschitz continuous in the direction $\theta$ for every $(\theta, \eta) \in \Lambda$. Necessary and sufficient conditions on $\Lambda$ are given for this assumption to imply each of the following: (1) that $f$ is Lipschitz continuous, and (2) that $f$ is continuous with modulus of continuity $\leq C \varepsilon|\log \varepsilon|$.
\end{abstract}

\section{Introduction}

Let us say that a function $f$ from $\mathrm{R}^{p}$ to $\mathrm{R}$ is continuous in the direction $\theta \in$ $\mathrm{R}^{p} \backslash\{0\}$ if the function $\mathrm{R} \ni t \mapsto f(x+t \theta)$ is continuous uniformly with respect to $x$ on compact sets. It is obvious that $f$ must be continuous if it satisfies $p$ conditions of this kind for a set of $\theta$-vectors that spans $\mathbf{R}^{p}$. However, to be able to conclude that two real-valued functions on $\mathrm{R}^{2}$ are continuous we do not need four conditions, but three conditions suffice. In fact, if $f$ is continous in the direction $(1,0), g$ is continous in the direction $(0,1)$, and $f+g$ is continuous in the direction $(1,1)$, then $f$ and $g$ must be continuous. This makes it natural to pose the following more general question.

Let $\Lambda$ be a finite set of pairs $(\theta, \eta) \in \mathrm{R}^{p} \times \mathrm{R}^{q}$ and let $f$ be a function from $\mathrm{R}^{p}$ to $\mathbf{R}^{q}$. Denote the inner product in $\mathrm{R}^{q}$ by $\langle\cdot, \cdot\rangle$. Assume that the real-valued function $x \mapsto\langle\eta, f\rangle(x)$ is continuous in the direction $\theta$ for every $(\theta, \eta) \in \Lambda$. Under what condition on $\Lambda$ does it follow that $f$ is continuous? The answer is given by Theorem 1.1. More precisely, if $\Lambda$ satisfies the condition $(\widetilde{A})$ and the function $x \mapsto\langle\eta, f\rangle(x)$ is continuous in the direction $\theta$ with modulus of continuity $\leq \sigma(t)$ for every $(\theta, \eta) \in \Lambda$, then $f$ must have modulus of continuity $\leq C t \int_{t}^{1} s^{-2} \sigma(s) d s$. In particular, if $\Lambda$ satisfies the condition $(\widetilde{A})$ and the function $x \mapsto\langle\eta, f\rangle(x)$ is Lipschitz continuous in the direction $\theta$ for every $(\theta, \eta) \in \Lambda$, then $f$ must have modulus of continuity $\leq C t \log (1 / t)$. The minimum number of elements of $\Lambda$ for $(\widetilde{A})$ to hold is $p+q-1$. This makes it natural to ask also under which condition on $\Lambda$ we can conclude that

\footnotetext{
* Supported by the International Science Programme (ISP), Uppsala University.

Received 24 November 2008, in final form 19 December 2011.
} 
the modulus of continuity of $f$ must be $\leq C \sigma(t)$. The answer to this question is also given by Theorem 1.1. The stronger conclusion requires at least $p q$ elements of $\Lambda$.

Those results were presented in [1] together with examples showing that the statements are sharp. Later it was shown in [2] that the results in [1] could be deduced from an extension to vector-valued functions of the theory of generalized moduli of continuity developed in [5], [4], and [3]. The purpose of this note is to give a considerably simpler proof of Theorem 1.1 than the ones given in [1] and [2]. We also give a complete proof of a more general statement that was announced without proof in [1].

Denote by $\Sigma$ the class of continuous, increasing, and subadditive functions from $\{t \in \mathrm{R} ; t>0\}$ into itself, tending to zero at the origin. If $\sigma \in \Sigma$ we denote by $K(\sigma)$ the set of (real-valued or vector-valued) functions $f$ on $\mathbf{R}^{p}$ that are continuous with modulus of continuity $\leq C \sigma(t)$ on compact sets, that is, for every compact set $K$ there exists a constant $C$ such that

$$
|f(x+y)-f(x)| \leq C \sigma(|y|), \quad x, x+y \in K .
$$

If $\theta \in \mathbf{R}^{p} \backslash\{0\}$ we denote by $K(\theta, \sigma)$ the set of functions that have modulus of continuity $\leq C \sigma(t)$ in the direction $\theta$, that is,

$$
|f(x+t \theta)-f(x)| \leq C \sigma(|t|), \quad x, x+t \theta \in K, \quad t \in \mathrm{R} .
$$

The set $\Lambda$ of pairs $(\theta, \eta) \in \mathbf{R}^{p} \times \mathbf{R}^{q}$ is said to satisfy condition (A) if the set of tensor products $\theta \otimes \eta$ for $(\theta, \eta) \in \Lambda$ spans $\mathrm{R}^{p} \otimes \mathrm{R}^{q}$. This is the same as saying that the set of $p \times q$ matrices $\left(\theta_{i} \eta_{j}\right)$ spans the $p q$-dimensional vector space $M(p, q)$ of all $p \times q$ matrices.

If we associate the $p \times q$ matrix $\left(a_{i j}\right)$ with the linear form $\left(\alpha_{i j}\right) \mapsto \sum \alpha_{i j} a_{i j}$ on $M(p, q)$, then the dual space of $M(p, q)$ will be identified with another copy of $M(p, q)$. The set $\Lambda$ is said to satisfy the condition $(\widetilde{A})$ if there is no rank one element of $M(p, q)$ that is orthogonal to all the matrices $\left(\theta_{i} \eta_{j}\right)$ for $(\theta, \eta) \in \Lambda$. Note that the condition (A) can be expressed by saying that there is no non-vanishing element of $M(p, q)$ that is orthogonal to all the matrices $\left(\theta_{i} \eta_{j}\right)$ for $(\theta, \eta) \in \Lambda$. In other words, $\Lambda$ satisfies $(\widetilde{A})$ if

$$
\begin{aligned}
& u \in \mathbf{R}^{p}, v \in \mathbf{R}^{q} \text { and }\langle u, \theta\rangle\langle v, \eta\rangle=0 \text { for all }(\theta, \eta) \in \Lambda \\
& \text { implies }|u||v|=0 .
\end{aligned}
$$

We also introduce two hull operations $P$ and $\widetilde{P}$ on subsets of $\mathrm{R}^{p} \times \mathrm{R}^{q}$ as follows. $P(\Lambda)$ is defined as the set of pairs $\left(\theta^{0}, \eta^{0}\right) \in \mathrm{R}^{p} \times \mathrm{R}^{q}$ such that $\theta^{0} \otimes \eta^{0}$ is in the linear hull of the set of all $\theta \otimes \eta$ such that $(\theta, \eta) \in \Lambda$. $\widetilde{P}(\Lambda)$ is the set of 
$\left(\theta^{0}, \eta^{0}\right)$ such that

$$
\begin{aligned}
& u \in \mathrm{R}^{p}, v \in \mathrm{R}^{q} \text { and }\langle u, \theta\rangle\langle v, \eta\rangle=0 \text { for all }(\theta, \eta) \in \Lambda \\
& \text { implies }\left\langle u, \theta^{0}\right\rangle\left\langle v, \eta^{0}\right\rangle=0 .
\end{aligned}
$$

We observe that condition (A) is equivalent to $P(\Lambda)=\mathrm{R}^{p} \times \mathrm{R}^{q}$ and condition $(\widetilde{A})$ is equivalent to $\widetilde{P}(\Lambda)=\mathrm{R}^{p} \times \mathrm{R}^{q}$.

If $\sigma \in \Sigma$ we define $\widetilde{\sigma}$ by

$$
\tilde{\sigma}(t)=t\left(\sigma(1)+\int_{\min (t, 1)}^{1} s^{-2} \sigma(s) d s\right), \quad t \geq 0 .
$$

If $\sigma$ and $\tau$ are in $\Sigma$ the expression $\sigma \prec \tau$ will mean that $\sigma(t) \leq C \tau(t)$ for $0<t<1$ and some $C$. If $\sigma \in \Sigma$, then $\widetilde{\sigma} \in \Sigma$ and $\sigma \prec \widetilde{\sigma}$. In fact, $\sigma(t) \leq \widetilde{\sigma}(t)$ for $t<1$, and the identity $t \widetilde{\sigma}^{\prime}(t)=\widetilde{\sigma}(t)-\sigma(t)$, valid for $t<1$, therefore implies $\tilde{\sigma}^{\prime}(t) \geq 0$ for $t<1$.

THEOREM 1.1. Let $f$ be a locally bounded function from $\mathrm{R}^{p}$ to $\mathrm{R}^{q}$ and let $\sigma \in \Sigma$. Let $\Lambda$ be a finite subset of $\mathrm{R}^{p} \times \mathrm{R}^{q}$ satisfying $(\widetilde{A})$ and assume that

$$
\langle\eta, f\rangle \in K(\theta, \sigma) \quad \text { for every } \quad(\theta, \eta) \in \Lambda .
$$

Then $f \in K(\widetilde{\sigma})$. Moreover, if $\Lambda$ satisfies $(A)$ and (1.3) holds, then $f \in K(\sigma)$. Conversely, if (1.3) implies $f \in K(\tau)$, then at least one of the following statements is true

$\Lambda$ satisfies $(A)$ and $\sigma \prec \tau$,

$\Lambda$ satisfies $(\widetilde{A})$ and $\widetilde{\sigma} \prec \tau$.

More generally, (1.3) implies $\left\langle\eta^{0}, f\right\rangle \in K\left(\theta^{0}, \tau\right)$ if and only if at least one of the following statements is true

$$
\begin{aligned}
& \left(\theta^{0}, \eta^{0}\right) \in P(\Lambda) \text { and } \sigma \prec \tau, \\
& \left(\theta^{0}, \eta^{0}\right) \in \widetilde{P}(\Lambda) \text { and } \widetilde{\sigma} \prec \tau .
\end{aligned}
$$

Let $M\left(\mathrm{R}^{p}\right)$ denote the space of measures on $\mathrm{R}^{p}$ with finite total mass, and let $\widehat{M}\left(\mathrm{R}^{p}\right)$ be the set of Fourier transforms of elements of $M\left(\mathrm{R}^{p}\right)$. The Fourier transform of $\mu \in M\left(\mathrm{R}^{p}\right)$ is defined by $\widehat{\mu}(\xi)=\left\langle\mu, e^{-i x \cdot \xi}\right\rangle=\int e^{-i x \cdot \xi} d \mu(x)$. Moreover we shall denote by $N_{\theta}\left(\mathrm{R}^{p}\right)$ the set of measures $\mu \in M\left(\mathrm{R}^{p}\right)$ for which

$$
\frac{\widehat{\mu}(\xi)}{\langle\theta, \xi\rangle} \in \widehat{M}\left(\mathrm{R}^{p}\right) .
$$

In [1] a similar role was played by the set $M_{\theta}\left(\mathrm{R}^{p}\right)$ of measures $\mu \in M\left(\mathrm{R}^{p}\right)$ for which the Fourier transform vanishes on the hyperplane perpendicular to $\theta$. It 
is clear that $N_{\theta}\left(\mathrm{R}^{p}\right) \subset M_{\theta}\left(\mathrm{R}^{p}\right)$, but the opposite inclusion is easily seen not to be true. (Take for instance $h(x)=\pi^{-1} /\left(1+x^{2}\right)-\psi(x)$, where $\psi \in L^{1}(\mathrm{R})$ is even, compactly supported, and has integral equal to 1 ; then $\widehat{h}(0)=0$ and $\widehat{h}(\xi)=e^{-|\xi|}-\widehat{\psi}(\xi)=-|\xi|+\mathcal{O}\left(|\xi|^{2}\right)$ as $|\xi| \rightarrow 0$, hence $\widehat{h}(\xi) / \xi$ is discontinuous at the origin and therefore cannot be in $\widehat{M}(\mathrm{R})$.) However, if $\mu \in M\left(\mathrm{R}^{p}\right)$ has compact support and $\widehat{\mu}(\xi)$ vanishes on the hyperplane $\langle\theta, \xi\rangle=0$, then $\mu \in N_{\theta}\left(\mathrm{R}^{p}\right)$ (c.f. Lemma 2.2). The fact that the measures constructed in Theorems 3 and 5 in [1] had compact support was the reason that the set $N_{\theta}\left(\mathrm{R}^{p}\right)$ was not needed in [1].

As in [1] we shall deduce Theorem 1.1 from two theorems on the representation of vector-valued measures as finite sums of measures of the form $\eta \otimes v_{\theta}$ where $v_{\theta} \in N_{\theta}\left(\mathrm{R}^{p}\right)$ and $(\theta, \eta) \in \Lambda$; see Section 2 . The novelties with our approach here relative to [1] are as follows. In the proof of Theorem 2.4 below the measure $\mu_{0}$ is written as a sum, $\mu_{0}=v_{0}+v_{1}$, of a measure $v_{0}$ whose Fourier transform has compact support and a measure $v_{1}$ whose Fourier transform vanishes in a neighborhood of the origin, and it is observed that the representation (2.1) is very easy to prove for $v_{0}$ and that (2.1) is an immediate consequence of Theorem 2.1 for $v_{1}$. The measures $\mu_{k}$ constructed in this way will not be compactly supported like those constructed in the proof of Theorem 3 in [1], but this is not needed for the proof of Theorem 1.1. Moreover, Theorem 2.1 is somewhat weaker than Theorem 5 in [1] in that $\widehat{\mu}_{0}$ is assumed to vanish in some neighborhood of the origin, but the proof is much simpler, the main new idea being the use of the special partition of unity (2.6). Finally, the measure $\mu$ in Lemma 3.3 with Fourier transform equal to 1 outside some compact set has replaced the iterated convolutions occurring in Theorem 5 of [1] (typically $q$-th order difference measures), and therefore Marchaud's inequality has been replaced by the easier and perhaps more fundamental Lemma 3.3 below.

The authors are indebted to Jan-Olov Strömberg for the proof of Lemma 3.1, which replaced an earlier, less elementary proof using results from [3].

\section{The submodule $J(\Lambda)$}

Denote by $M\left(\mathrm{R}^{p}\right)^{q}$ the set of $q$-tuples of measures in $M\left(\mathrm{R}^{p}\right)$. It is the tensor product over $\mathrm{R}$ of the vector spaces $\mathrm{R}^{q}$ and $M\left(\mathrm{R}^{p}\right)$. The tensor product $\eta \otimes \mu$ can be defined as the $q$-tuple $\left(\eta_{1} \mu, \ldots, \eta_{q} \mu\right) \in M\left(\mathrm{R}^{p}\right)^{q}$, and from now on it will simply be denoted $\eta \mu$.

Since $M\left(\mathrm{R}^{p}\right)$ is a ring under convolution, it is clear that $M\left(\mathrm{R}^{p}\right)^{q}$ is a module over the ring $M\left(\mathrm{R}^{p}\right)$. We shall denote by $J(\Lambda)$ the set of element of $M\left(\mathrm{R}^{p}\right)^{q}$ of the form

$$
\sum_{k=1}^{m} \eta^{k} \mu_{k}
$$


where $\mu_{k} \in N_{\theta^{k}}\left(\mathrm{R}^{p}\right)$ and $\left(\theta^{k}, \eta^{k}\right) \in \Lambda$. Since $N_{\theta^{k}}\left(\mathrm{R}^{p}\right)$ is an ideal in $M\left(\mathrm{R}^{p}\right)$ it is clear that $J(\Lambda)$ is a submodule of $M\left(\mathrm{R}^{p}\right)^{q}$. The set of Fourier transforms of elements of $J(\Lambda)$ will be denoted $\widehat{J}(\Lambda)$.

THEOREM 2.1. Assume that $\left(\theta^{0}, \eta^{0}\right) \in \widetilde{P}(\Lambda)$ and that $\mu_{0} \in M\left(\mathrm{R}^{p}\right)$ satisfies $\widehat{\mu}_{0}(\xi)=0$ when $\left|\left\langle\theta^{0}, \xi\right\rangle\right|<\varepsilon$ for some $\varepsilon>0$. Then $\eta^{0} \mu_{0} \in J(\Lambda)$. Moreover, if $\Lambda$ satisfies $(\widetilde{A})$ and $\widehat{\mu}_{0}(\xi)=0$ in some neighborhood of the origin, then $\eta^{0} \mu_{0} \in J(\Lambda)$ for each $\eta^{0} \in \mathrm{R}^{q}$.

It is easy to see that the first statement of Theorem 2.1 implies the second. In fact, if $\widehat{\mu}_{0}(\xi)=0$ in a neighborhood of the origin then we can find $\varepsilon>0$ and $\mu_{j}, j=1, \ldots, p$, such that $\widehat{\mu}_{j}(\xi)=0$ for $\left|\xi_{j}\right|<\varepsilon$ and $\mu_{0}=\sum \mu_{j}$. Applying the first statement of Theorem 2.1 to $\left(\theta^{0}, \eta^{0}\right)$ where $\eta^{0}$ is arbitrary and $\theta^{0}$ is the $j$ :th basis vector in $\mathrm{R}^{p}$ we obtain $\eta^{0} \mu_{j} \in J(\Lambda)$, hence $\eta^{0} \mu_{0} \in J(\Lambda)$.

The following simple lemma will be needed below.

LeMma 2.2. Let $\mu \in M(\mathrm{R})$ and assume that $\widehat{\mu}(\xi)=0$ in some neighborhood of the origin. Then $\widehat{\mu}(\xi) / \xi$ is in $\widehat{M}(\mathrm{R})$. More generally, assume that $\widehat{\mu}(\xi)$ is twice continuously differentiable in some neighborhood of the origin and that $\widehat{\mu}(0)=0$. Then $\widehat{\mu}(\xi) / \xi$ is in $\widehat{M}(\mathrm{R})$.

Proof. Let $\delta>0$ be so small that supp $\widehat{\mu} \subset\{|\xi|>\delta\}$ and choose $\chi \in$ $C^{\infty}(\mathrm{R})$ such that $\chi(\xi)=0$ in for $|\xi|<\delta / 2$ and $\chi(\xi)=1$ for $|\xi|>\delta$. Then $\widehat{\mu}(\xi)=\chi(\xi) \widehat{\mu}(\xi)$, so it is enough to prove that $\chi(\xi) / \xi$ is in $\widehat{M}(\mathrm{R})$. Since $\chi(\xi) / \xi$ is in $L^{2}(\mathrm{R})$ there exists $h \in L^{2}(\mathrm{R}) \subset L_{\mathrm{loc}}^{1}(\mathrm{R})$ so that $\widehat{h}(\xi)=\chi(\xi) / \xi$. And to see that $h \in L^{1}(\mathrm{R}) \subset M(\mathrm{R})$ it is enough to observe that the second derivative of $\chi(\xi) / \xi$ is in $L^{1}(\mathrm{R})$, hence $x^{2} h(x)$ is bounded. This proves the first statement. To prove the second statement we note that $(1-\chi(\xi)) \widehat{\mu}(\xi)$ is twice continuously differentiable, compactly supported, and vanishes at $\xi=0$, hence $(1-\chi(\xi)) \widehat{\mu}(\xi) / \xi$ is continuously differentiable and compactly supported, and it is well known that such a function is in $\widehat{L^{1}}(\mathrm{R}) \subset \widehat{M}(\mathrm{R})$.

The next lemma gives reformulations of the conditions $(\widetilde{A})$ and $\left(\theta^{0}, \eta^{0}\right) \in$ $\widetilde{P}(\Lambda)$. Let $\mathscr{L}$ denote linear hull.

Lemma 2.3. Let $\left(\theta^{k}, \eta^{k}\right), k=1,2, \ldots m$, be the elements of $\Lambda$. Denote an arbitrary subset of $\{1,2, \ldots, m\}$ by $E$ and its complement by $E^{\prime}$. Then $\left(\theta^{0}, \eta^{0}\right) \in \widetilde{P}(\Lambda)$ if and only if

for each $E$ at least one of the following statements is true:

$$
\theta^{0} \in \mathscr{L}\left(\left\{\theta^{k} ; k \in E\right\}\right), \quad \eta^{0} \in \mathscr{L}\left(\left\{\eta^{k} ; k \in E^{\prime}\right\}\right) .
$$

In particular $\Lambda$ satisfies $(\widetilde{A})$ if and only if (2.2) is true for all $\left(\theta^{0}, \eta^{0}\right) \in \mathbf{R}^{p} \times \mathbf{R}^{q}$, 
which is equivalent to

for each $E$ at least one of the following statements is true:

$$
\mathscr{L}\left(\left\{\theta^{k} ; k \in E\right\}\right)=\mathrm{R}^{p}, \quad \mathscr{L}\left(\left\{\eta^{k} ; k \in E^{\prime}\right\}\right)=\mathrm{R}^{q} .
$$

Proof. If (2.2) is false, then there exist $u \in \mathrm{R}^{p}$ and $v \in \mathrm{R}^{q}$ such that $\left\langle u, \theta^{k}\right\rangle=0$ for all $k \in E,\left\langle v, \eta^{k}\right\rangle=0$ for all $k \in E^{\prime}$, and $\left\langle u, \theta^{0}\right\rangle \neq 0,\left\langle v, \eta^{0}\right\rangle \neq$ 0 . But this means that $\left\langle u, \theta^{k}\right\rangle\left\langle v, \eta^{k}\right\rangle=0$ for all $k$ and $\left\langle u, \theta^{0}\right\rangle\left\langle v, \eta^{0}\right\rangle \neq 0$, in other words, $\left(\theta^{0}, \eta^{0}\right) \notin \widetilde{P}(\Lambda)$. Conversely, if $\left(\theta^{0}, \eta^{0}\right) \notin \widetilde{P}(\Lambda)$, then there exists $(u, v)$ so that $\langle u, \theta\rangle\langle v, \eta\rangle=0$ for all $(\theta, \eta) \in \Lambda$ but $\left\langle u, \theta^{0}\right\rangle\left\langle v, \eta^{0}\right\rangle \neq 0$. Choosing $E$ as the set of $k$ for which $\left\langle u, \theta^{k}\right\rangle=0$ we have $\left\langle v, \eta^{k}\right\rangle=0$ for all $k \in E^{\prime}$, which shows that (2.2) is false and completes the proof of the first statement. The second statement is proved similarly.

It follows immediately from Lemma 2.3 that $\Lambda$ must have at least $p+q-1$ elements, if $(\widetilde{A})$ holds.

Since we shall need certain standard cut-off functions many times, we define them here once and for all in order to avoid repetitions. We shall denote by $\psi$ and $\chi$ two functions in $C^{\infty}(\mathrm{R})$ such that

$$
\begin{aligned}
\psi(t) & =1 \quad \text { for }|t|<1 / 2, \\
\psi(t) & =0 \text { for }|t|>1, \\
\text { and } \quad \chi^{2} & =1-\psi(t)
\end{aligned}
$$

Proof of Theorem 2.1. Although it would be sufficient to prove the first statement, we begin by proving the second statement in order to simplify the exposition. We first claim that it is enough to prove that we can find measures $v_{k} \in N_{\theta^{k}}\left(\mathrm{R}^{p}\right)$ such that

$$
\eta^{0}=\sum_{k=1}^{m} \eta^{k} \widehat{v}_{k}(\xi), \quad|\xi|>B,
$$

for some sufficiently large $B$. To see this note first that $\widehat{\mu}_{0}(\xi)=\chi(|\xi| / \varepsilon) \widehat{\mu}_{0}(\xi)$ if $\varepsilon$ is small enough, hence it is enough to prove that $\eta^{0} \chi(|\xi| / \varepsilon)$ is in $\widehat{J}(\Lambda)$. Since $N_{\theta}\left(\mathrm{R}^{p}\right)$ is obviously invariant under the variable transformation $\xi \mapsto A \xi$ for every $A>0$ and every $\theta$, the same must be true for $\widehat{J}(\Lambda)$. But (2.5) implies that $\eta^{0} \chi(|\xi| / 2 B)$ is in $\widehat{J}(\Lambda)$, hence $\eta^{0} \chi(|\xi| / \varepsilon)$ is in $\widehat{J}(\Lambda)$, and the claim is proved.

It is easy to establish (2.5) locally in conic subsets of $\mathrm{R}^{p}$ as follows. Assume that $(\widetilde{A})$ holds and let $\xi^{0}$ be an arbitrary element of $\mathrm{R}^{p} \backslash\{0\}$. Let $E$ be the set 
of $k$ for which $\left\langle\theta^{k}, \xi^{0}\right\rangle \neq 0$. It follows from Lemma 2.3 that the set of $\eta^{k}$ for which $k \notin E$ must span $\mathrm{R}^{q}$. Hence we can find constants $b_{k}$ such that

$$
\eta^{0}=\sum_{k \notin E} b_{k} \eta^{k}
$$

Let $\chi(t) \in C^{\infty}(\mathrm{R})$ be the function defined by (2.4) and set

$$
h(\xi)=\prod_{k \in E} \chi\left(\left\langle\theta^{k}, \xi\right\rangle\right) .
$$

By Lemma 2.2 we have $h \in \widehat{N}_{\theta^{k}}\left(\mathrm{R}^{p}\right)$ for each $k \in E$. By the choice of $E$ we have $h\left(\lambda \xi^{0}\right)=1$ if $\lambda>0$ is sufficently large, and by continuity the same is true for $\xi$ in some neighborhood of $\xi^{0}$. Taking $\widehat{v}_{k}(\xi)=b_{k} h(\xi)$ we have obtained (2.5) in a conic neighborhood of $\xi^{0}$. We can cover $\mathrm{R}^{p} \backslash\{0\}$ by a finite number of open conic sets $V_{i}$ and for each $V_{i}$ construct $v_{k}^{i}$ such that (2.5) holds in $V_{i} \cap\{\xi ;|\xi|>B\}$ for some $B$. It is now natural to try to piece together those measures $v_{k}^{i}$ by choosing a partition of unity $\phi_{i}(\xi)$ on the unit sphere $S^{p-1} \subset \mathrm{R}^{p}$ with supp $\phi_{i} \subset V_{i} \cap S^{p-1}$ and choose $v_{k}$ by

$$
\widehat{v}_{k}(\xi)=\sum_{i} \phi_{i}(\xi /|\xi|) \widehat{v}_{k}^{i}(\xi)
$$

The problem is that the functions $\phi(\xi /|\xi|)$, homogeneous of degree zero, are in general not in $\widehat{M}\left(\mathrm{R}^{p}\right)$. Therefore we need a different partition of unity in $\mathrm{R}^{p}$, especially adapted to this problem.

Let $\psi$ and $\chi$ be the functions defined by (2.4). If $\left(\theta^{k}, \eta^{k}\right), k=1, \ldots, m$, are the elements of $\Lambda$ we set $\psi_{k}(\xi)=\psi\left(\left\langle\theta^{k}, \xi\right\rangle\right), \chi_{k}(\xi)=\chi\left(\left\langle\theta^{k}, \xi\right\rangle\right)$, and write

$$
1=\prod_{k=1}^{m}\left(\psi_{k}(\xi)+\chi_{k}(\xi)\right) .
$$

Expanding the product we obtain a sum of $2^{m}$ terms,

$$
1=\sum_{E} \phi_{E}(\xi)
$$

where

$$
\phi_{E}(\xi)=\prod_{k \in E} \psi_{k}(\xi) \prod_{k \in E^{\prime}} \chi_{k}(\xi),
$$

and the sum in (2.6) is taken over all subsets $E$ of $\{1,2, \ldots, m\}$. We have to show that $\eta^{0} \phi_{E}(\xi)$ is in $\widehat{J}(\Lambda)$ for each $E$. 
If the set of $\theta^{k}$ with $k \in E$ spans $\mathrm{R}^{p}$, then the support of $\prod_{k \in E} \psi_{k}(\xi)$, and hence the support of $\phi_{E}$, is compact, so we can disregard those terms. Thus consider an arbitrary term $\phi_{E}$ where the set of $\theta^{k}$ with $k \in E$ does not span $\mathrm{R}^{p}$. By Lemma 2.3 the set of $\eta^{k}$ for $k \in E^{\prime}$ must then span $\mathrm{R}^{q}$. Choosing $b_{k}$ so that $\eta^{0}=\sum_{k \in E^{\prime}} b_{k} \eta^{k}$ and multiplying with $\phi_{E}(\xi)$ we obtain

$$
\eta^{0} \phi_{E}(\xi)=\sum_{k \in E^{\prime}} b_{k} \eta^{k} \phi_{E}(\xi)
$$

We claim that $\phi_{E}(\xi)$ is in $\widehat{N}_{\theta^{k}}\left(\mathrm{R}^{p}\right)$ for each $k \in E^{\prime}$. But this is clear because $\phi_{E}(\xi) /\left\langle\theta^{k}, \xi\right\rangle$ is a product of $\psi_{j}(\xi)$ for $j \in E$ and $\chi_{j}(\xi)$ for $j \in E^{\prime}, j \neq k$, and the factor

$$
\chi\left(\left\langle\theta^{k}, \xi\right\rangle\right) /\left\langle\theta^{k}, \xi\right\rangle
$$

which is in $\widehat{M}\left(\mathrm{R}^{p}\right)$ by Lemma 2.2 . In this way we also see that $\phi_{E}(\xi)=0$ for $\left|\left\langle\theta^{k}, \xi\right\rangle\right|<1 / 2$. This completes the proof of the second statement of Theorem 2.1.

To prove the first statement we observe first by reasoning as above that it is enough to prove that $\eta^{0} \chi\left(\left\langle\theta^{0}, \xi\right\rangle / B\right)$ is in $\widehat{J}(\Lambda)$ for sufficiently large $B$. Consider $\eta^{0} \phi_{E}(\xi) \chi\left(\left\langle\theta^{0}, \xi\right\rangle / B\right)$ for an arbitrary $E$. If $\eta^{0} \in \mathscr{L}\left(\left\{\eta^{k} ; k \in E^{\prime}\right\}\right)$ we can choose numbers $b_{k}$ such that $\eta^{0}=\sum_{k \in E^{\prime}} b_{k} \eta^{k}$. Since $\phi_{E}(\xi)$ contains the factor $\chi\left(\left\langle\theta^{k}, \xi\right\rangle\right)$ for every $k \in E^{\prime}$, we can write

$$
\eta^{0} \phi_{E}(\xi) \chi\left(\left\langle\theta^{0}, \xi\right\rangle / B\right)=\sum_{k \in E^{\prime}} b_{k} \eta^{k} \phi_{E}(\xi) \chi\left(\left\langle\theta^{0}, \xi\right\rangle / B\right),
$$

and observe that $\phi_{E}(\xi)$ is in $\widehat{N}_{\theta^{k}}\left(\mathrm{R}^{p}\right)$ for every $k \in E^{\prime}$, so the expression (2.8) is in $\widehat{J}(\Lambda)$ in this case. If $\eta^{0} \notin \mathscr{L}\left(\left\{\eta^{k} ; k \in E^{\prime}\right\}\right)$, then by Lemma 2.3 we must have $\theta^{0} \in \mathscr{L}\left(\left\{\theta^{k} ; k \in E\right\}\right)$. But in that case the function $\phi_{E}(\xi) \chi\left(\left\langle\theta^{0}, \xi\right\rangle / B\right)$ must vanish if $B$ is large enough, because the support of $\phi_{E}$ is contained in $\left|\left\langle\theta^{0}, \xi\right\rangle\right| \leq A$ for some $A$. In fact $\operatorname{supp} \phi_{E} \subset\left\{\xi ;\left|\left\langle\theta^{k}, \xi\right\rangle\right| \leq 1\right\}$ for every $k \in E$, and if $\theta^{0}=\sum_{k \in E} a_{k} \theta^{k}$ we have $\left|\left\langle\theta^{0}, \xi\right\rangle\right| \leq \sum\left|a_{k}\right|\left\langle\theta^{k}, \xi\right\rangle\left|\leq \sum\right| a_{k} \mid$ for every $\xi \in \operatorname{supp} \phi_{E}$, which proves the claim and finishes the proof of the theorem.

We now turn to the consequences of the stronger hypothesis $(A)$.

THeOREm 2.4. Assume that $\Lambda$ satisfies $(A)$ and let $\mu_{0} \in N_{\theta^{0}}\left(\mathrm{R}^{p}\right)$ for some $\theta^{0} \in \mathrm{R}^{p} \backslash\{0\}$. Then $\eta^{0} \mu_{0} \in J(\Lambda)$. More generally, if $\mu_{0} \in N_{\theta^{0}}\left(\mathrm{R}^{p}\right)$ and $\left(\theta^{0}, \eta^{0}\right) \in P(\Lambda)$, then $\eta^{0} \mu_{0} \in J(\Lambda)$.

Proof. We begin by proving the first statement. Take a smooth function $\phi(\xi)$ supported in $|\xi|<2$ and equal to 1 in $|\xi| \leq 1$ and decompose $\mu_{0}$ as $\mu_{0}=$ $v_{0}+v_{1}$ by taking $\widehat{v}_{0}(\xi)=\phi(\xi) \widehat{\mu}_{0}(\xi)$ and $v_{1}=\mu_{0}-v_{0}$. The fact that $v_{1} \in J(\Lambda)$ 
follows from Theorem 2.1 since $\widehat{v}_{1}(\xi)=0$ in a neighborhood of the origin. It remains to consider $v_{0}$. Now, since $\Lambda$ satisfies $(A)$, there exist constants $c_{k}$ and $\left(\theta^{k}, \eta^{k}\right) \in \Lambda, k=1, \ldots, m$, such that $\eta^{0} \otimes \theta^{0}=\sum_{k=1}^{m} c_{k} \eta^{k} \otimes \theta^{k}$, or equivalently,

$$
\eta^{0}\left\langle\theta^{0}, \xi\right\rangle=\sum_{k=1}^{m} c_{k} \eta^{k}\left\langle\theta^{k}, \xi\right\rangle, \quad \xi \in \mathrm{R}^{p} .
$$

Since $\mu_{0} \in N_{\theta^{0}}\left(\mathrm{R}^{p}\right)$, we have $\widehat{\mu}_{0}(\xi)=\left\langle\theta^{0}, \xi\right) \widehat{\lambda}(\xi)$ for some $\lambda \in M\left(\mathrm{R}^{p}\right)$, hence by (2.9)

$$
\begin{aligned}
& \eta^{0} \widehat{v}_{0}(\xi)=\eta^{0} \widehat{\mu}_{0}(\xi) \phi(\xi)=\eta^{0}\left\langle\theta^{0}, \xi\right\rangle \widehat{\lambda}(\xi) \phi(\xi) \\
& =\sum_{k=1}^{m} c_{k} \eta^{k}\left\langle\theta^{k}, \xi\right\rangle \widehat{\lambda}(\xi) \phi(\xi)=\sum_{k=1}^{m} \eta^{k} \widehat{\mu}_{k}(\xi)
\end{aligned}
$$

with $\widehat{\mu}_{k}(\xi)=c_{k}\left\langle\theta^{k}, \xi \backslash \widehat{\lambda}(\xi) \phi(\xi)\right.$. Since $\phi$ has compact support it is clear that $\mu_{k} \in M\left(\mathrm{R}^{p}\right)$ and that $\mu_{k} \in N_{\theta^{k}}\left(\mathrm{R}^{p}\right)$, which completes the proof of the first statement.

To prove the second statement assume that $\left(\theta^{0}, \eta^{0}\right) \in P(\Lambda)$ and that $\mu_{0} \in$ $N_{\theta^{0}}\left(\mathrm{R}^{p}\right)$. Decomposing $\mu_{0}$ by $\mu_{0}=v_{0}+v_{1}$, where $\widehat{\nu}_{0}(\xi)=\widehat{\mu}_{0} \psi\left(\left\langle\theta^{0}, \xi\right\rangle\right)$ and $\psi \in C^{\infty}(\mathrm{R})$ is defined by (2.4), we see by the first part of Theorem 2.1 that $\eta^{0} v_{1} \in J(\Lambda)$, so it is sufficient to study $v_{0}$. And since $\widehat{v}_{0}(\xi)$ is divisible by $\left\langle\theta^{0}, \xi\right\rangle \psi\left(\left\langle\theta^{0}, \xi\right\rangle\right)$, it is enough to prove that $\left\langle\theta^{0}, \xi\right\rangle \psi\left(\left\langle\theta^{0}, \xi\right\rangle\right)$ is in $\widehat{J}(\Lambda)$.

By the assumption we have (2.9) and multiplying this equation by $\psi\left(\left\langle\theta^{0}, \xi\right\rangle\right)$ we obtain

$$
\eta^{0}\left\langle\theta^{0}, \xi\right\rangle \psi\left(\left\langle\theta^{0}, \xi\right\rangle\right)=\sum_{k} c_{k} \eta^{k}\left\langle\theta^{k}, \xi\right\rangle \psi\left(\left\langle\theta^{0}, \xi\right\rangle\right) .
$$

The difficulty is that the functions $\left\langle\theta^{k}, \xi\right\rangle \psi\left(\left\langle\theta^{0}, \xi\right\rangle\right)$ are not in $\widehat{M}\left(\mathrm{R}^{p}\right)$ because they are in general not even bounded. Choose measures $\rho_{k}$ by

$$
\widehat{\rho}_{k}(\xi)=c_{k}\left\langle\theta^{k}, \xi\right\rangle \psi\left(\left\langle\theta^{k}, \xi\right\rangle\right) \psi\left(\left\langle\theta^{0}, \xi\right\rangle\right) .
$$

Clearly $\rho_{k} \in N_{\theta^{k}}\left(\mathrm{R}^{p}\right)$. If we insert $1=\psi\left(\left\langle\theta^{k}, \xi\right\rangle\right)+\chi\left(\left\langle\theta^{k}, \xi\right\rangle\right)$ in the $k$ th term in (2.10), we obtain

$$
\eta^{0}\left\langle\theta^{0}, \xi\right\rangle \psi\left(\left\langle\theta^{0}, \xi\right\rangle\right)=\sum_{k} \eta^{k} \widehat{\rho}_{k}(\xi)+F(\xi),
$$

where

$$
F(\xi)=\psi\left(\left\langle\theta^{0}, \xi\right\rangle\right) \sum_{k} c_{k} \eta^{k}\left\langle\theta^{k}, \xi\right\rangle \chi\left(\left\langle\theta^{k}, \xi\right\rangle\right) .
$$

It remains to show that $F(\xi)$ is in $\widehat{J}(\Lambda)$. 
From (2.11) we see that each component of the (vector-valued) function $F(\xi)$ is in $\widehat{M}\left(\mathrm{R}^{p}\right)$ (although the individual terms in (2.12) are not). Thus, if we take a subset $\left\{\eta^{k} ; k \in E_{0}\right\}$ of the set of all the $\eta^{k}$ that forms a basis for the vector space spanned by all the $\eta^{k}$, then we can write

$$
F(\xi)=\sum_{j \in E_{0}} b_{j}(\xi) \eta^{j},
$$

where $b_{j}(\xi)$ are uniquely determined functions that belong to $\widehat{M}\left(\mathrm{R}^{p}\right)$. Indeed, each $b_{j}(\xi)$ must be a linear combination of the components of $F(\xi)$. Note that the functions $b_{j}(\xi)$ depend on our choice of basis. Our problem is that we want each $b_{j}(\xi)$ to vanish on $\left\langle\theta^{j}, \xi\right\rangle=0$. To achieve this we have to split $F(\xi)$ into a number of terms by means of a partition of unity, just as we did before, and choose different bases for the different terms.

It will be convenient to use the partition of unity $1=\sum_{E} \phi_{E}(2 \xi)$. The reason for the factor 2 is that $\psi(2 t) \chi(t)=0$, so that

$$
\psi\left(2\left\langle\theta^{k}, \xi\right\rangle\right) \chi\left(\left\langle\theta^{k}, \xi\right\rangle\right)=0 \quad \text { for all } k
$$

It will be enough to prove that $F(\xi) \phi_{E}(2 \xi)$ is in $\widehat{J}(\Lambda)$ for an arbitrary $E$.

Fix $E$ and consider $F(\xi) \phi_{E}(2 \xi)$. Because of (2.13) all terms in (2.12) with $k \in E$ will drop out. We therefore choose $E_{0}$ consisting only of vectors $\eta^{j}$ with $j \in E^{\prime}$, and write

$$
F(\xi) \phi_{E}(2 \xi)=\sum_{j \in E_{0}} b_{j}(\xi) \eta^{j}
$$

with $E_{0} \subset E^{\prime}$. But $\phi_{E}(\xi)$ is defined so that it contains every $\chi\left(\left\langle\theta^{j}, \xi\right\rangle\right)$ with $j \in E^{\prime}$ as factor, hence in particular all $\chi\left(\left\langle\theta^{j}, \xi\right\rangle\right)$ with $j \in E_{0}$. As we saw above, each $b_{j}(\xi)$ is a linear combination of the components of the function $F(\xi)$. Hence each $b_{j}(\xi)$ belongs to $\widehat{N}_{\theta^{j}}\left(\mathrm{R}^{p}\right)$, and the proof is complete.

\section{Proof of Theorem 1.1}

If $\mu \in M\left(\mathrm{R}^{p}\right)$ and $t>0$ we define the measure $\mu_{t}$ by $\widehat{\mu_{t}}(\xi)=\widehat{\mu}(t \xi)$. For a function $k \in L^{1}\left(\mathrm{R}^{p}\right) \subset M\left(\mathrm{R}^{p}\right)$ this means that $k_{t}(x)=t^{-p} k(x / t)$. In deducing Theorem 1.1 from Theorem 2.1 and Theorem 2.4 we shall need to estimate the supremum norm of convolutions $\mu_{t} * f$ in terms of the modulus of continuity $\omega(f, t)$ and vice versa. This makes it natural to introduce the so-called generalized modulus of continuity $\omega_{\mu}(f, t)$ of a continuous and compactly supported function $f$ with respect to a measure $\mu$ as follows:

$$
\omega_{\mu}(f, t)=\sup \left\{\left|\mu_{s} * f(x)\right| ; x \in \mathbf{R}^{p},|s|<t\right\}
$$


Estimates of the kind we need were called comparison theorems for generalized moduli of continuity in [3] and [4] and were studied in great detail there.

If $\mu=v * \rho$, then it is obvious that

$$
\omega_{\mu}(f, t) \leq C \omega_{\nu}(f, t)
$$

with $C=\|\rho\|_{M}$. The set of continuous and compactly supported functions on $\mathrm{R}^{p}$ will be denoted by $C_{c}\left(\mathrm{R}^{p}\right)$.

Lemma 3.1. Assume that the measure $\mu \in M(\mathrm{R})$ satisfies the condition

$$
|x| \mu \in M(\mathrm{R}),
$$

and that $\mu$ has mean zero, that is $\widehat{\mu}(0)=\langle\mu, 1\rangle=0$. Then

$$
\omega_{\mu}(f, t) \leq C \omega(f, t), \quad f \in C_{c}(\mathrm{R}),
$$

with $C=\int(1+|x|)|d \mu(x)|$.

Proof. Since $\langle\mu, 1\rangle=0$ we can write

$$
\begin{aligned}
\mu_{t} * f(x) & =\int f(x-y) d \mu_{t}(y)=\int f(x-t y) d \mu(y) \\
& =\int(f(x-t y)-f(x)) d \mu(y) .
\end{aligned}
$$

Set $\omega(f, t)=\sigma(t)$. Since $\sigma(t)$ is subadditive and increasing it follows that

$$
\left|\mu_{t} * f(x)\right| \leq \int_{\mathrm{R}} \sigma(|y t|)|d \mu(y)| \leq \int_{\mathrm{R}}(1+|y|) \sigma(t)|d \mu(y)| \leq C \sigma(t),
$$

which proves the assertion.

Lemma 3.2. Let $\theta \in \mathrm{R}^{p} \backslash\{0\}$ and assume $f \in C_{c}\left(\mathrm{R}^{p}\right) \cap K(\theta, \sigma)$ and $\mu \in N_{\theta}\left(\mathrm{R}^{p}\right)$. Then

$$
\omega_{\mu}(f, t) \leq C \sigma(t)
$$

Proof. After a rotation of the coordinate system we may assume that $\theta=$ $(1,0, \ldots, 0)$. The assumption $\mu \in N_{\theta}\left(\mathrm{R}^{p}\right)$ then means that $\widehat{\mu}(\xi) / \xi_{1}$ is in $\widehat{M}\left(\mathrm{R}^{p}\right)$. Take $\psi$ as in (2.4) and decompose $\mu$ by writing $\mu=\mu_{0}+\mu_{1}$ where $\widehat{\mu}_{0}(\xi)=\widehat{\mu}(\xi) \psi\left(\xi_{1}\right)$. Denote the difference measure $\delta_{(1,0, \ldots, 0)}-\delta_{(0, \ldots, 0)}$ by $\Delta_{1}$. Then

$$
\frac{\widehat{\mu}_{0}(\xi)}{\widehat{\Delta}_{1}(\xi)}=\frac{\widehat{\mu}(\xi)}{\xi_{1}} \cdot \frac{\psi\left(\xi_{1}\right)}{\left(e^{-i \xi_{1}}-1\right) / \xi_{1}}
$$


is in $\widehat{M}\left(\mathrm{R}^{p}\right)$, since the last factor is a smooth compactly supported function of $\xi_{1}$. Thus $\mu_{0}=\Delta_{1} * \tau$ for some $\tau \in M\left(\mathrm{R}^{p}\right)$, hence

$$
\omega_{\mu_{0}}(f, t) \leq C \omega_{\Delta_{1}}(f, t) \leq C_{1} \sigma(t)
$$

with $C=\|\tau\|_{M}$. It remains to estimate $\omega_{\mu_{1}}(f, t)$. Since $\widehat{\mu}_{1}(\xi)=0$ for $\left|\xi_{1}\right|<$ $1 / 2$ we can write $\widehat{\mu}_{1}(\xi)=\chi\left(2 \xi_{1}\right) \widehat{\mu}_{1}(\xi)$, where $\chi$ is the function defined by (2.4), hence we have $\omega_{\mu_{1}}(f, t) \leq \omega_{\rho}(f, t)\left\|\mu_{1}\right\|_{M}$, if $\rho$ is the measure in $M\left(\mathrm{R}^{p}\right)$ defined by $\widehat{\rho}(\xi)=\chi\left(2 \xi_{1}\right)$. It is therefore enough to estimate $\omega_{\rho}(f, t)$ in terms of $\omega_{\Delta_{1}}(f, t)$. This is essentially a one-variable problem, since $\rho$ and $\Delta_{1}$ are measures supported on the $x_{1}$-axis. Considering $\rho$ as a measure in $M(\mathrm{R})$ it is in fact equal to the Dirac measure plus a Schwartz function, so it certainly satisfies the condition (3.1). The estimate $\omega_{\rho}(f, t) \leq C \omega_{\Delta_{1}}(f, t)$ therefore follows from Lemma 3.1. The proof is complete.

The next lemma is a converse estimate for a very special class of measures $\mu$. The case that we shall need is when $\widehat{\mu}(\xi)=0$ in some neighborhood of the origin, and this case immediately implies the more general statement of the lemma.

Lemma 3.3. Let $\mu \in M(\mathrm{R})$ be a measure such that $\widehat{\mu}(\xi)=1$ outside some compact set. Then

$$
\omega(f, t) \leq C\left(\omega_{\mu}(f, t)+t \int_{t}^{\infty} s^{-2} \omega_{\mu}(f, s) d s\right),
$$

$f \in C_{c}(\mathrm{R}), t>0$, hence $\omega(f, t) \leq C \widetilde{\omega}_{\mu}(f, s)$.

Proof. If $\widehat{\mu}(0) \neq 0$, then $\omega_{\mu}(f, t)$ in general does not tend to zero as $t \rightarrow 0$, so the statement is empty in this case. If $\widehat{\mu}(\xi)$ vanishes at the origin of order at most 1 in the sense that $\xi / \widehat{\mu}(\xi)$ is locally in $\widehat{L^{1}}(\mathrm{R})$ near the origin, then the stronger conclusion $\omega(f, t) \leq C \omega_{\mu}(f, C t)$ holds [3, Corollary 2.3]. The interesting case is therefore when $\widehat{\mu}(\xi)$ vanishes at the origin of higher order than 1. The statement of the lemma is a special case of Corollary 2.4 in [3], but the case considered here is much simpler because of the assumption that $\widehat{\mu}(\xi)=1$ for large $|\xi|$. We therefore give the short proof here.

By scaling we see that we may assume that $\widehat{\mu}(\xi)=1$ for $|\xi|>1 / 4$. Let $\psi$ be the function defined by (2.4). Decompose the difference measure $\Delta$ by writing $\Delta=v_{0}+v_{1}$ with $\widehat{v}_{0}(\xi)=\psi(\xi) \widehat{\Delta}(\xi)$. Then $\widehat{v}_{1}=\widehat{v}_{1} \widehat{\mu}$, so it is enough to estimate $\omega_{\nu_{0}}(f, t)$. Since $\widehat{\Delta}(\xi)=e^{-i \xi}-1$ we can write $\widehat{v}_{0}(\xi)=\psi(\xi) \xi h(\xi)$, where $h(\xi)$ has compact support and is equal to $\left(e^{-i \xi}-1\right) / \xi$ on the support of $\psi$, hence $h \in L^{1}(\mathrm{R})$. Therefore it is enough to estimate $\omega_{\rho}(f, t)$ where $\widehat{\rho}(\xi)=\xi \psi(\xi)$. Set $\phi(\xi)=\psi(\xi)-\psi(2 \xi)$. Then $\psi(\xi)=\sum_{k=0}^{\infty} \phi\left(2^{k} \xi\right)$ for 
$\xi \neq 0$, and hence

$$
\xi \psi(\xi)=\sum_{k=0}^{\infty} \xi \phi\left(2^{k} \xi\right)=\sum_{k=0}^{\infty} 2^{-k} 2^{k} \xi \phi\left(2^{k} \xi\right)=\sum_{k=0}^{\infty} 2^{-k} \phi_{1}\left(2^{k} \xi\right)
$$

where $\phi_{1}(\xi)=\xi \phi(\xi)$. Since $\widehat{\mu}(\xi)=1$ on the support of $\phi_{1}$, we have $\phi_{1}(\xi)=$ $\widehat{\mu}(\xi) \phi_{1}(\xi)$ and hence $\omega_{\lambda}(f, t) \leq C \omega_{\mu}(f, t)$ if $\lambda \in L^{1}(\mathrm{R})$ is the function defined by $\widehat{\lambda}(\xi)=\phi_{1}(\xi)$. The only non-trivial step is to estimate $\omega_{\rho}(f, t)$ in terms of $\omega_{\lambda}(f, s)$. To do this we replace $\xi$ by $t \xi$ in (3.3) and take inverse Fourier transforms to obtain $\rho_{t}=\sum 2^{-k} \lambda_{t 2^{k}}$, and hence

$$
\omega_{\rho}(f, t) \leq \sum_{k=0}^{\infty} 2^{-k} \omega_{\lambda}\left(f, 2^{k} t\right)
$$

It is an elementary fact that this implies

$$
\omega_{\rho}(f, t) \leq t \int_{t}^{\infty} s^{-2} \omega_{\lambda}(f, s) d s .
$$

In fact for any non-negative increasing function $\varphi$ we have

$$
t \int_{t 2^{k}}^{t 2^{k+1}} s^{-2} \varphi(s) d s \geq t \varphi\left(t 2^{k}\right) \int_{t 2^{k}}^{t 2^{k+1}} s^{-2} d s=2^{-k-1} \varphi\left(t 2^{k}\right) .
$$

Recalling that $\omega_{\nu_{0}}(f, t) \leq C \omega_{\rho}(f, t)$ and that $\omega_{\lambda}(f, t) \leq C \omega_{\mu}(f, t)$ we can now conclude (3.2).

Proof of TheOREM 1.1. Using a partition of unity it is easy to see that it is sufficient to consider the case when $f$ has compact support. We first prove the assertions under the additional assumption that $f$ is continuous. Assume now that $\left(\theta^{0}, \eta^{0}\right) \in P(\Lambda)$ and that $\left\langle\eta^{k}, f\right\rangle \in K\left(\theta^{k}, \sigma\right)$ for every $\left(\theta^{k}, \eta^{k}\right) \in \Lambda$. We have to prove that $\left\langle\eta^{0}, f\right\rangle \in K\left(\theta^{0}, \sigma\right)$. Choose $\mu_{0}=\delta_{\theta^{0}}-\delta_{(0, \ldots, 0)}$. It is easy to see that $\mu_{0} \in N_{\theta^{0}}\left(\mathrm{R}^{p}\right)$, because the measure $\nu \in M(\mathrm{R})$ defined by $\delta_{0}-\delta_{1}$ has the property that $\widehat{v}(\lambda) / \lambda$ is in $\widehat{M}(\mathrm{R})$ by Lemma 2.2 . We can therefore apply Theorem 2.4 and obtain measures $\mu_{k} \in N_{\theta^{k}}\left(\mathrm{R}^{p}\right)$ such that

$$
\eta^{0} \mu_{0}=\sum_{k} \eta^{k} \mu_{k}
$$

holds with $\mu_{k} \in N_{\theta^{k}}\left(\mathrm{R}^{p}\right)$. Applying Lemma 3.2 to the function $\mathrm{R} \ni t \mapsto$ $\left\langle\eta^{k}, f\right\rangle\left(x+t \theta^{k}\right)$ we can infer from the assumption $\left\langle\eta^{k}, f\right\rangle \in K\left(\theta^{k}, \sigma\right)$ that $\omega_{\mu_{k}}\left(\left\langle\eta^{k}, f\right\rangle, t\right) \leq C \sigma(t)$ for every $k$. The identity (3.4) now shows that $\omega_{\mu_{0}}\left(\left\langle\eta^{0}, f\right\rangle, t\right) \leq C \sigma(t)$ with a new constant $C$, which proves the assertion. 
Assume next that $\left(\theta^{0}, \eta^{0}\right) \in \widetilde{P}(\Lambda)$. Define $\mu_{0}$ by $\widehat{\mu}_{0}(\xi)=\chi\left(\left\langle\theta^{0}, \xi\right\rangle\right)$. By Theorem 2.1 we can find $\mu_{k} \in N_{\theta^{k}}\left(\mathrm{R}^{p}\right)$ such that (3.4) holds. Reasoning as in the previous paragraph we can conclude that $\omega_{\mu_{0}}\left(\left\langle\eta^{0}, f\right\rangle, t\right) \leq C \sigma(t)$. Finally, by Lemma 3.3 we can infer from here that $\left\langle\eta^{0}, f\right\rangle \in K\left(\theta^{0}, \widetilde{\sigma}\right)$, which completes the proof for the case when $f$ is assumed to be continuous.

To prove those statements without the à priori assumption that $f$ is continuous it is sufficient to apply (3.4) to a sequence of regularizations of $f$ and use standard arguments.

The converse statement is easy to prove using ideas from [1] as follows. It is obvious that (1.3) cannot imply $\left\langle\eta^{0}, f\right\rangle \in K\left(\theta^{0}, \tau\right)$ unless $\sigma \prec \tau$. If $\left(\theta^{0}, \eta^{0}\right) \notin \widetilde{P}(\Lambda)$ we take $u \in \mathrm{R}^{p}, v \in \mathrm{R}^{q}$ such that $\langle u, \theta\rangle\langle v, \eta\rangle=0$ for all $(\theta, \eta) \in \Lambda,\left\langle u, \theta^{0}\right\rangle\left\langle v, \eta^{0}\right\rangle \neq 0$, and choose $f(x)=v h(\langle u, x\rangle)$, where $h$ is a discontinuous function of one variable. Then $t \mapsto\langle\eta, f(x+t \theta)\rangle$ is constant for every $(\theta, \eta) \in \Lambda$ but $t \mapsto\left\langle\eta^{0}, f\left(x+t \theta^{0}\right)\right\rangle$ is not continuous, so (1.3) does not imply $\left\langle\eta^{0}, f\right\rangle \in K\left(\theta^{0}, \tau\right)$ for any $\tau$. In the case $\left(\theta^{0}, \eta^{0}\right) \in \widetilde{P}(\Lambda)$ but $\left(\theta^{0}, \eta^{0}\right) \notin P(\Lambda)$ we consider only $\sigma(t)=t$; for general $\sigma$ one can reason as in [1], p. 21. Assume $\left(\theta^{0}, \eta^{0}\right) \notin P(\Lambda)$ and choose a linear operator $B: \mathrm{R}^{p} \rightarrow \mathrm{R}^{q}$ such that $\langle\eta, B \theta\rangle=0$ for all $(\theta, \eta) \in \Lambda$ but $\left\langle\eta^{0}, B \theta^{0}\right\rangle \neq 0$. Let $f(x)=B x \log |x|$. Then it is easy to see that $t \mapsto\left\langle\eta^{0}, f\left(x+t \theta^{0}\right)\right\rangle$ has bounded derivative, hence $\langle\eta, f\rangle$ is in $K(\theta, \sigma)$, for every $(\theta, \eta) \in \Lambda$, but $\left\langle\eta^{0}, f\right\rangle$ is in $K\left(\theta^{0}, \tau\right)$ only if $\widetilde{\sigma}(t) \sim t|\log t| \prec \tau$.

\section{REFERENCES}

1. Boman, J., Partial regularity of mappings between Euclidean spaces, Acta Math. 119 (1967), $1-25$.

2. Boman, J., Comparison theorems for generalized moduli of continuity. Vector valued measures, pp. 38-52 in: Construction theory of functions of several variables, Lecture Notes in Math. 571, Springer, Berlin 1977.

3. Boman, J., Equivalence of generalized moduli of continuity, Ark. Mat. 18 (1980), 73-100.

4. Boman, J., and Shapiro, H. S., Comparison theorems for a generalized modulus of continuity, Ark. Mat. 9 (1971), 91-116.

5. Shapiro, H. S., A Tauberian theorem related to approximation theory, Acta Math. 120 (1968), 279-292.

DEPARTMENT OF MATHEMATICS

UNIVERSITY OF BUEA

CAMEROON

E-mail: dieu_agb@yahoo.co.uk
DEPARTMENT OF MATHEMATICS STOCKHOLM UNIVERSITY SE-10691 STOCKHOLM SWEDEN

E-mail: jabo@math.su.se 